To appear 2006.09.01 in the Astrophysical Journal

\title{
A Transiting Planet of a Sun-like Star
}

\author{
P. R. McCullough ${ }^{1,2}$, J. E. Stys ${ }^{1}$, Jeff A. Valenti ${ }^{1}$, C. M. Johns-Krull ${ }^{3,4}$, K. A. Janes ${ }^{5}$,

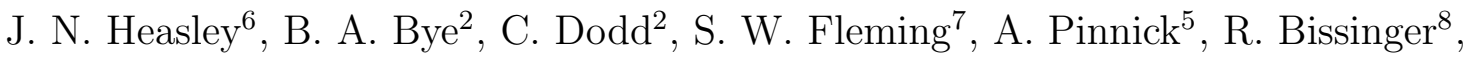 \\ B. L. Gary ${ }^{9}$, P. J. Howell ${ }^{5}$, T. Vanmunster ${ }^{10}$ \\ pmcc@stsci.edu
}

\begin{abstract}
A planet transits an $11^{\text {th }}$ magnitude, G1V star in the constellation Corona Borealis. We designate the planet $\mathrm{XO}-1 \mathrm{~b}$, and the star, $\mathrm{XO}-1$, also known as GSC 02041-01657. XO-1 lacks a trigonometric distance; we estimate it to be $200 \pm 20$ pc. Of the ten stars currently known to host extrasolar transiting planets, the star XO-1 is the most similar to the Sun in its physical characteristics: its radius is $1.0 \pm 0.08 \mathrm{R}_{\odot}$, its mass is $1.0 \pm 0.03 \mathrm{M}_{\odot}, \mathrm{V} \sin i<3 \mathrm{~km} \mathrm{~s}^{-1}$, and its metallicity $[\mathrm{Fe} / \mathrm{H}]$ is $0.015 \pm 0.04$. The orbital period of the planet $\mathrm{XO}-1 \mathrm{~b}$ is $3.941534 \pm 0.000027$ days, one of the longer ones known. The planetary mass is $0.90 \pm 0.07 \mathrm{M}_{\mathrm{J}}$, which is marginally larger than that of other transiting planets with periods between 3 and 4 days. Both the planetary radius and the inclination are functions of the spectroscopically determined stellar radius. If the stellar radius is $1.0 \pm 0.08 R_{\odot}$, then the planetary radius is $1.30 \pm 0.11 R_{J}$ and the inclination of the orbit is $87.7 \pm 1.2^{\circ}$. We have demonstrated a productive international
\end{abstract}

${ }^{1}$ Space Telescope Science Institute, 3700 San Martin Dr., Baltimore MD 21218

${ }^{2}$ University of Illinois, Urbana, IL 61801

${ }^{3}$ Dept. of Physics and Astronomy, Rice University, 6100 Main Street, MS-108, Houston, TX 77005

${ }^{4}$ Visiting Astronomer, McDonald Observatory, which is operated by the University of Texas at Austin.

${ }^{5}$ Boston University, Astronomy Dept., 725 Commonwealth Ave., Boston, MA 02215

${ }^{6}$ University of Hawaii, Inst. for Astronomy, 2680 Woodlawn Dr., Honolulu, HI 96822-1839

${ }^{7}$ Dept. of Astronomy, University of Florida, 211 Bryant Space Science Center, Gainesville, FL 32611

${ }^{8}$ Racoon Run Observatory, Pleasanton, CA

${ }^{9}$ Hereford Arizona Observatory, 5320 E. Calle Manzana, Hereford, AZ 85615

${ }^{10} \mathrm{CBA}$ Belgium Observatory, Walhostraat 1A, B-3401 Landen, Belgium 
collaboration between professional and amateur astronomers that was important to distinguishing this planet from many other similar candidates.

Subject headings: binaries: eclipsing - planetary systems - stars: individual (GSC 02041-01657) - techniques: photometric - techniques: radial velocities

\section{Introduction}

We know of five planets that transit stars brighter than $\mathrm{V}=12$ : HD209458b, TrES-1b, HD 149026b, HD 189733b, and (now) XO-1b (Charbonneau et al. 2000 and Henry et al. 2000; Alonso et al. 2004; Sato et al. 2005; Bouchy et al. 2005a, and this work, respectively report the discoveries). ${ }^{1}$ Observations of planetary transits of fainter stars still permit a rather precise measure of the relative size of a star and its planet, but the brighter stars provide adequate flux to enable interesting follow-up observations, including observations of atmospheric composition (Charbonneau et al. 2002; Vidal-Madjar et al. 2004), exosphere extent (Vidal-Madjar et al. 2003), and infrared emission (Charbonneau et al. 2005; Deming et al. 2005). The five remaining systems, discovered by OGLE (e.g. Bouchy et al. 2005b), are too faint $(\mathrm{V} \sim 15)$ for such studies. The XO project aims to find planets transiting stars sufficiently bright to enable interesting follow up studies (McCullough et al. 2005). ${ }^{2}$

In addition to atmospheric studies of planets, transits around bright stars also provide the best constraints on planetary interior models, which currently cannot explain the observations (Konacki et al. 2005). They also can provide an empirical determination of stellar limb darkening and the frequency and contrast of star spots (Silva 2003). Transits provide simple 1-dimensional imaging of the stellar photospheres, because an inverted transit light curve represents a 1-dimensional trace through the 2-dimensional convolution of the star's brightness distribution with a uniform disk equal in radius to the planet. In an analogous manner, eclipses in principle can provide the brightness distribution on the face of the planet as the star acts as a knife edge cutting across the disk of the planet during ingress or egress. Herein, we use "transit" to describe a planet passing in front of a star, and "eclipse" to describe a star passing in front of a planet or another star.

\footnotetext{
${ }^{1}$ Alonso et al (2004) referred to both the planet and its host star as "TrES-1," but for clarity we prefer the trailing "b" for the planets.

${ }^{2}$ This paper includes data taken on the Haleakala summit maintained by the University of Hawaii, the Lowell Observatory, the McDonald Observatory of The University of Texas at Austin, and four backyard observatories.
} 
Comparison studies will become increasingly interesting and important as different methods of discovering transiting extrasolar planets produce additional systems. Some radial-velocity surveys (Robinson et al. 2006; da Silva et al. 2006) have a selection bias for high metallicity stars, in order to increase the fraction of observed stars that have hot Jupiters (Valenti \& Fischer 2005). Photometric surveys for transits are biased toward large depths of the transits (i.e large planets and/or small stars) and short periods, both of which increase detectability.

One of the challenges of transit surveys such as the XO project is distinguishing the stars with transiting planets from those without. Initial discovery of candidate planets requires observing thousands of stars, many hundreds of times each, with $\sim 1 \%$ or better photometry per epoch. A light curve consistent with a transiting planet more often is due to a grazing eclipsing binary star, a triple star system, or a stellar companion that is of planetary size but not of planetary mass (Brown 2003).

Whereas high-resolution spectroscopy is required to measure a planetary companion's mass, either moderate-resolution spectroscopy or high-fidelity photometry in many cases can distinguish the grazing eclipsing binary stars and the triple stars (Section 4.2) from transiting planets. Practical considerations may determine which is the preferred method (spectroscopy or photometry). A spectrum will often reveal a double-lined eclipsing binary immediately, and a pair of spectra separated by mere hours can reveal the large amplitude $\left(\mathrm{km} \mathrm{s}^{-1}\right)$ radial velocity variation of a single-lined eclipsing binary (or multiple) star system. Such spectra can be obtained with moderate aperture (e.g. 2-m diameter) telescopes and with little regard for the ephemeris of the transits. On the other hand, precise photometry of transits demands that the candidate be observed at specific and predictable times that nevertheless are inconveniently infrequent for observing in a "traditional" manner by an astronomer who must wait months to observe in hopes that during the week or two that he or she is allocated on a telescope, and the sky is clear, and the sun is down, and the star is at low to moderate zenith angle, the planet will transit. Faced with such prospects, some astronomers have deployed dedicated robotic telescopes for photometric follow up (e.g. Sleuth of O'Donovan, Charbonneau, \& Kotredes 2004; TopHat of Bakos et al. 2006a).

We chose a different tactic: we formed a collaboration of professional and amateur astronomers. The high-quality photometry produced by amateur astronomers of the transits of HD 209458 and Tres-1 (Naeye 2004) immediately after their announcements by professional astronomers proved that amateur astronomers can produce photometry of individual stars that exceeds the quality obtainable by an instrument (such as XO) that was designed to survey thousands of stars quickly. The XO P.I. recruited four amateur astronomers (T. V., R. B., B. G., and P. H.) to assist with photometric follow up of candidates identified in the 
XO data. Important criteria in selecting these individuals were 1) the observatories should be distributed in longitude, permitting a given transit to have a high probability of being observed quickly, 2) each member should have exclusive access to a CCD-equipped telescope and the expertise and software to produce differentially-calibrated time-series photometry, 3) the telescope(s) should be capable of unattended operation, in order to reduce attrition even though only a small fraction of targets would be genuine planets, and 4) internet access and a team-oriented attitude. Henden (2006) describes other amateur-professional collaborations; one related to transiting planets is transitsearch.org (Seagroves et al. 2003).

Whereas some have advocated dispersing survey instruments longitudinally around the globe (e.g. Alonso et al. 2004), the example of XO-1b demonstrates the effectiveness of a survey instrument such as XO on a single excellent site such as Haleakala, especially if it is coupled to a longitudinally dispersed network of observatories capable of subsequent study of candidates individually at specific times. We hope that as the XO Extended Team (E.T.) of advanced amateur astronomers grows in members, its capabilities and its value to extrasolar planet discovery will grow geometrically.

\section{Observations}

\subsection{XO Project Photometry}

McCullough et al. (2005) describe the instrumentation, operation, analysis, and preliminary results of the XO project. ${ }^{3}$ In summary, the XO observatory monitored tens of thousands of bright $(\mathrm{V}<12)$ stars twice every ten minutes on clear nights for more than 2 months per season of visibility for each particular star, over the period September 2003 to September 2005. From our analysis of more than 3000 observations per star, we identified XO-1 (Figure 1) as one of dozens of stars with light curves suggestive of a transiting planet. With the XO cameras on Haleakala, we observed three transits of XO-1 in 2004 and one in 2005, on Julian dates 2453123, 2453127, 2453143, and 2453454. From the survey photometry of XO-1 (Figure 2), which has a nominal standard deviation of $0.8 \%$ or 8 mmag per observation, we determined a preliminary light curve and ephemeris, which we used to schedule observations of higher quality with other telescopes, as described in the next section.

\footnotetext{
${ }^{3}$ The analysis of XO photometry is incomplete, so a statistical analysis would be premature and would tend to underestimate the efficiency for discovering similar transiting planets within the data.
} 


\subsection{Additional Photometry}

In June and July 2005, we observed XO-1 from three observatories in North America and one in Europe. Telescopes of $\gtrsim 0.2-\mathrm{m}$ diameter have adequate sensitivity for these purposes, and a network of them is well suited to observe candidates with known positions and ephemerides. A few days after XO-1 was selected from the thousands of stars XO monitors, the XO E.T. obtained light curves from independent telescopes sufficient to discern a steep ingress and egress flanking a relatively wide flat bottom (Figure 3). With angular resolution $\sim 20$ times finer than the survey cameras', the E.T. observations reduced the likelihood that XO-1 was a dilute triple system, i.e. one star with a fainter, eclipsing binary nearby, which can mimic a transiting planet (Charbonneau et al. 2004).

On the basis of the E.T. light curves of 2005, we acquired spectra of XO-1 as described in the next section. After the first few spectra showed XO-1b has substellar mass, we obtained additional photometry (Figure 4) with the PRISM reimager (Janes et al. 2005) in $\mathrm{B}, \mathrm{V}$, and R filters on the 1.8-m Perkins telescope at Lowell observatory, and with the E.T. telescopes. The photometry from the 1.8-m telescope was acquired in a repeated exposure sequence of nine 5-s $\mathrm{R}$ images, three 10-s V images, and one 30-s B image; the $\mathrm{R}$ and $\mathrm{V}$ data were averaged into 0.002-day bins. The E.T. R data were averaged into 0.006-day bins. Binning permitted outlier rejection and empirical estimation of the noise by the scatter of the individual observations, which is helpful in cases such as this in which scintillation can be a significant and unpredictable component to noise.

We estimate all-sky photometric $\mathrm{B}, \mathrm{V}, \mathrm{R}_{\mathrm{C}}$, and $\mathrm{I}_{\mathrm{C}}$ magnitudes for $\mathrm{XO}-1$ and several

nearby reference stars (Figure 1 and Table 1) calibrated using two Landolt areas (Landolt 1992). Using a 0.35-meter telescope on photometric nights 2006 February 25 and 2006 March 14, we measured the fluxes of 28 and 22 Landolt stars, respectively at an air mass similar to that for $\mathrm{XO}-1$ and established the zero points of the instrumental magnitudes and transformation equations for the color corrections for each filter and the CCD. The $\mathrm{R}_{\mathrm{C}}$ magnitudes for XO-1 and 8 reference stars differed on the two dates by a standard deviation of $0.005 \mathrm{mag}$. The $\mathrm{B}, \mathrm{V}, \mathrm{R}_{\mathrm{C}}$, and $\mathrm{I}_{\mathrm{C}}$ absolute photometric accuracies are $0.04,0.03,0.02$, and 0.03 mag r.m.s., including both the formal error and an estimated systematic error. The Tycho magnitudes for XO-1 listed in Table 2 transform (via Table 2 of Bessel 2000) to Johnson $V=11.25$, i.e. $0.06 \mathrm{mag}(2-\sigma)$ fainter than our estimate. 


\subsection{Spectroscopy}

In order to measure the mass ratio of the system and to determine the characteristics of the host star, we obtained spectra of XO-1 with two-dimensional cross-dispersed echelle spectrographs (Tull et al. 1995; Tull 1998) at the coude focus of the 2.7-m Harlan J. Smith (HJS) telescope and via a fiber optic on the 11-m Hobby-Eberly Telescope (HET). Both telescopes are located at McDonald Observatory. The HJS spectra were obtained in a traditionally-scheduled manner, and the HET spectra were queued. We used an iodine gas cell on the HET, but not on the HJS telescope. We obtained one or two high-resolution $(\lambda / \Delta \lambda \approx 60000)$ spectra on each of ten nights. We extracted the two-dimensional echelle spectra using procedures described in Hinkle et al. (2000).

\section{Analysis}

\subsection{Ephemeris}

We refined our estimate of XO-1b's orbital period $P$ from modeling eight pairs of transits chosen as follows: each of four transits observed by the E. T. on 2005 June 23, 2005 July 01, 2005 July 05, and 2005 July 12 (Figure 3) was paired with two observations of the 2006 March 14 transit (Figure 4). We solved for the best-fitting period for each pair of transits. The mean orbital period, $P$ is 3.941534 days; the $1-\sigma$ dispersion is 0.000027 days. The heliocentric Julian date of minimum light (also mid-transit) is

$$
t_{m . l .}=t_{c}+P \times E
$$

where $\mathrm{E}$ is an integer, and $t_{c}=2453808.9170 \pm 0.0011$ (HJD). Identical parameters are listed in Table 3 and certainly will be refined in subsequent publications. In estimating the parameters of Equation 1, we used only the photometry of the eight pairs of transits mentioned previously. The XO survey photometry gives similar parameters but with larger uncertainties, as expected because the survey data (by design) have poorer photometric precision and cadence than the E. T. data.

\subsection{Radial Velocity Measurements}

We measured XO-1's radial velocities with respect to the topocentric frame using iodine absorption lines superposed on the HET spectra of XO-1. We modeled the extracted spectra using high-resolution spectra $\left(\lambda / \Delta \lambda \approx 10^{6}\right)$ of the Sun and the Earth's atmosphere (Wallace, 
Hinkle, \& Livingston 1998) and the HET iodine gas cell (Cochran 2000). Using an IDL ${ }^{4}$ implementation of Nelder and Mead's (1965) downhill simplex $\chi^{2}$ minimization algorithm, "Amoeba," we adjusted parameters of our model to fit the observations. The model includes convolution of our input spectra with a best-fitting Voigt profile to approximate the (slightly non-Gaussian) line-spread-function of the instrument. The free parameters of our model are a continuum normalization factor, the radial velocity of the star, the radial velocity of the iodine lines (which represent instrumental deviations from their expected zero velocity with respect to the observatory), and an exponent (optical depth scale factor) that "morphs" the depths of the lines, as an arbitrary method of adjusting the solar spectrum to that of XO-1. Due to the iodine absorption, we could not estimate the continuum level by interpolating between local maxima in the spectrum, so instead we solved for the continuum iteratively, as required to improve the fit between our model and the observations. In the manner described above, for each $\sim 15 \AA$ section of each individual spectrum within the region of the recorded spectrum with significant iodine absorption, $5100-5700 \AA$, we estimated the radial velocity of the star. From the approximately normal distribution of the resulting radial velocity estimates for each epoch, we calculated the mean radial velocity and its uncertainty. We transformed our measured radial velocities to the barycentric frame of the solar system and phased them to the ephemeris known from the transits (Figure 5 and Table 4).

The 1- $\sigma$ internal errors of the radial velocities from the iodine spectra are $\sim 15 \mathrm{~m} \mathrm{~s}^{-1}$ per epoch (i.e. per average of two 15-minute exposures) with the 11-m HET and $\sim 65 \mathrm{~m} \mathrm{~s}^{-1}$ per 15- or 20-minute exposure with the 2.7-m HJS telescope. We used only the iodine-calibrated radial velocities to determine the amplitude of the radial velocity variation, assumed to be sinusoidal and phased with the transits. An eccentricity approximately equal to zero is expected theoretically (Bodenheimer et al. 2001) and is consistent with the radial velocities (Figure 5c). The maximum likelihood radial velocity semi-amplitude $\mathrm{K}=116 \pm 9 \mathrm{~m} \mathrm{~s}^{-1}$.

\subsection{Spectroscopically-Derived Stellar Properties and Planetary Mass}

An initial estimate of the spectral type of XO-1 was obtained using the line depth ratio method developed by Gray \& Johanson (1991). The spectral type is estimated by measuring the depth of the V I $6251.83 \AA$ line divided by the depth of the adjacent Fe I $6252.57 \AA$ line. The 4 spectra from the HJS telescope were summed together and the individual line depths were measured by fitting a Gaussian function to each line. The resulting line ratio is 0.14, which corresponds to a G1 star (Table 1 of Gray \& Johanson 1991). Differences in metallicity

\footnotetext{
${ }^{4}$ IDL is a registered trademark of Research Systems, Inc.
} 
can affect these results (Gray 1994), so we estimate an uncertainty of one spectral subclass for the spectral type of X0-1. These published line depth ratios are for dwarf stars, and Gray \& Brown (2001) show that there is a gravity dependence to the spectral type classification that results from this ratio. We estimate the luminosity class $\mathrm{V}$ by examining the gravitysensitive $\mathrm{Na} \mathrm{D}$ doublet and the $\mathrm{Mg}$ b triplet. Below we estimate the $\log g=4.53 \pm 0.065$ $\mathrm{cm} \mathrm{s}^{-2}$, whereas early G-type luminosity class III giants have $\log g \approx 2.5 \mathrm{~cm} \mathrm{~s}^{-2}$, which produces substantially narrower Na D profiles (Gray 1992). Our final spectral type estimate for $\mathrm{XO}-1$ is $\mathrm{G} 1 \mathrm{~V}$.

We used the software package SME (Valenti \& Piskunov 1996) to fit each of the four spectra of XO-1 from the HJS telescope with synthetic spectra. We used the methodology of Valenti \& Fischer (2005), including their minor corrections to match the Sun and remove abundance trends with temperature (negligible in this case). Because of gaps between echelle orders, the HJS spectra are missing the wavelength intervals $6000-6123 \AA$ and $6113-6118 \AA$, which were included in the Valenti \& Fischer analysis. These wavelength intervals are also missing from our extracted HET spectra because the relevant echelle orders span the gap between the two detectors.

We averaged our SME results for the four HJS spectra, obtaining the parameter values in Table 5. Each value in the last column of the table, labeled "Precision" because systematic uncertainties are not included, is the standard deviation of the four measurements divided by $\sqrt{3}$ to yield the formal uncertainty in the mean. The median value of each derived parameter (not given) differs from the mean by less than the uncertainty in the mean. The final row in the table gives [Si/Fe], which Valenti \& Fischer (2005) used as a proxy for alpha-element enrichment, when interpolating isochrones.

Figure 6 shows XO-1's spectrum in the region of the $\mathrm{Mg}$ b triplet, which is the dominant spectroscopic constraint on gravity. These three Mg lines also have a significant impact on the global $[\mathrm{M} / \mathrm{H}]$ parameter, which is used to scale solar abundances for all elements other than Na, Si, Ti, Fe, and Ni. The unidentified absorption lines at $5171 \AA$ and in the wing of the $6020 \AA$ line are common in stars of this type (Valenti \& Fisher 2005) so presumably are unrelated to the presence of a hot Jupiter.

Following the methodology of Fischer \& Valenti (2005), we interpolated Yonsei-Yale $\left(\mathrm{Y}^{2}\right)$ isochrones (Demarque et al. 2004) to determine probability distribution functions for the mass, radius, gravity, and age of XO-1. The trigonometric parallax of XO-1 is unknown, so we initially assumed distances of 180,200 , and 220 pc with an adopted uncertainty of $10 \mathrm{pc}$ in each case (which affects the width of the resulting distribution functions). We measured a V magnitude of $11.19 \pm 0.03$ for XO-1 (Table 2) so XO-1 would be at 190 pc, if it has the same intrinsic brightness as the Sun. 
We used our spectroscopic effective temperature, spectroscopic gravity, and an assumed distance to derive a bolometric correction by interpolating the "high temperature" table from VandenBerg \& Clem (2003). We combined the bolometric correction with the observed V-band magnitude to determine stellar luminosity. Then we used the stellar luminosity and our spectroscopic effective temperature, iron abundance, and alpha-element enrichment to interpolate the $\mathrm{Y}^{2}$ isochrones to produce the probability distribution functions in Figure 7.

As expected, the most probable mass, radius, gravity, and age are essentially solar for an assumed distance of 200 pc. If we assume that XO- 1 is at $180 \mathrm{pc}$ instead, then the most probable age drops to $2 \mathrm{Gyr}$, which is unlikely given how slowly XO- 1 rotates $(\mathrm{V} \sin i<3$ $\mathrm{km} \mathrm{s}^{-1}$ ). If we assume XO-1 is at a distance of $220 \mathrm{pc}$, then the gravity from stellar structure models deviates significantly from the gravity we derived spectroscopically. Thus, we assume in subsequent analysis that XO-1 is at a distance of 200 pc, but clearly the trigonometric parallax needs to be measured.

Assuming a distance of $200 \pm 10$ pc yields a $68 \%$ confidence limit of $1.00 \pm 0.025$ for $\mathrm{M} / \mathrm{M}_{\odot}, 1.00 \pm 0.05$ for $\mathrm{R} / \mathrm{R}_{\odot}$, and 2.5-6.6 Gyr for the age (Figure 7). The stellar mass combined with our measured semi-amplitude $\mathrm{K}=116 \pm 9 \mathrm{~m} \mathrm{~s}^{-1}$ yields a mass of $0.90 \pm 0.07$ $\mathrm{M}_{\mathrm{J}}$ for the planet XO-1b. Within the broader range of distances of $200 \pm 20 \mathrm{pc}$ (Table 6 ), the light curves and the spectroscopy produce consistent estimates of the stellar radius (Figure 8). Based upon XO-1's proper motion, radial velocity, and distance, its velocity vector with respect to the local standard of rest is $(\mathrm{u}, \mathrm{v}, \mathrm{w})=(8,9,23) \mathrm{km} \mathrm{s}^{-1}$, which is consistent with the kinematics of a G-dwarf with an age of a few Gyr (Wyse \& Gilmore 1995).

\subsection{Light Curve Modeling and Planetary Radius}

We modeled the observed transit light curves using the algorithms of Mandel \& Agol (2002) and adopt their notation. For the star, we interpolate quadratic limb darkening coefficients from Claret (2000) for the filter bandpasses: $\mathrm{B}\left(\gamma_{1}=0.639 ; \gamma_{2}=0.179\right)$, V $\left(\gamma_{1}=0.437 ; \gamma_{2}=0.295\right)$, or $\mathrm{R}\left(\gamma_{1}=0.339 ; \gamma_{2}=0.324\right)$, based upon our spectroscopic gravity and effective temperature and assuming solar metallicity, which is consistent with our spectroscopic determination (Table 5). In addition to the two limb darkening coefficients, parameters of a model light curve include the mass $M_{*}$ of the star, the size ratio $p=r_{p} / r_{*}$ of the radius of the planet $r_{p}$ to the radius $r_{*}$ of the star, the inclination $i$ and the angular frequency $\omega$ of the orbit, and the heliocentric epoch of the transit's center, $t_{c}$. Note that the shape depends on the size ratio $p=r_{p} / r_{*}$ and not the two radii separately.

Of the seven input parameters $\left(\gamma_{1}, \gamma_{2}, M_{*}, p, i, \omega, t_{c}\right)$ the most precisely measured are $\omega$ 
and $t_{c}$. As previously noted, we estimated $\gamma_{1}$ and $\gamma_{2}$ from the spectroscopically-determined $\log g$ and $T_{\text {eff }}$. Our derivation of the other parameters is similar to that of Brown et al. (2001). We begin by adopting the stellar mass $M_{*}$ and its uncertainty from the previous section. Combining the mass $M_{*}$ and the angular frequency $\omega$ with an assumption of a circular orbit gives the velocity of the planet passing in front of the star. Combining that velocity with the observed duration of the transit (from 2 nd contact to 3 rd contact) gives a chord length for the transit across the star. The half chord length $L$ must be less than or equal to the radius of the star, i.e. $L \leq r_{*}$. The stellar radius $r_{*}$ also has an upper bound from the light curve: for a transit of a given $L$, the larger $r_{*}$ becomes, the longer will be the ingress and the egress, as the transit becomes more grazing. Thus, the duration from 1st to 2 nd contacts (or 3 rd to 4 th) creates an upper bound, $r_{*} \lesssim 1.3 \mathrm{R}_{\odot}$, whereas the duration from 2 nd to 3 rd contacts creates a lower bound, $r_{*} \gtrsim 0.9 \mathrm{R}_{\odot}$.

For rhetorical purposes we have discussed the transits as if the contacts are distinct features in the light curves, although with the precision of our photometry, they are not. Instead, the model light curves become too narrow compared to the observations if $r_{*} \lesssim$ $0.9 \mathrm{R}_{\odot}$, and the models become too V-shaped to match the "steep-edged" U-shape of the observations if $r_{*} \gtrsim 1.3 \mathrm{R}_{\odot}$. In between these extremes, $r_{*}$ and $i$ are anti-correlated: increases in $r_{*}$ can be accommodated by decreases in $i$, and vice versa:

$$
\begin{aligned}
i & =\cos ^{-1} \sqrt{\theta_{\odot}^{2} \hat{P}^{-4 / 3} \hat{\rho}^{-2 / 3}-\pi^{2} q^{2}} \\
& \approx \frac{\pi}{2}-\sqrt{\theta_{\odot}^{2} \hat{P}^{-4 / 3} \hat{\rho}^{-2 / 3}-\pi^{2} q^{2}}
\end{aligned}
$$

where $\theta_{\odot}=1 \mathrm{R}_{\odot} / 1 \mathrm{~A} . \mathrm{U} .=4.65 \times 10^{-3} \mathrm{rad}, \hat{P}$ is the planet's orbital period in years, $\hat{\rho}$ is the dimensionless mean density of the star in solar units i.e. $\hat{\rho}=\left(m_{*} / \mathrm{M}_{\odot}\right)\left(r_{*} / \mathrm{R}_{\odot}\right)^{-3}$, and we determine the scalar $q$ so as to match the relation between each best-fitting inclination $i$ for each assumed value for $\hat{\rho}$. Geometrically, $q$ is the dimensionless duty cycle of the transit, i.e. the duration of the transit divided by the period, where the transit's duration is defined as the interval between the two moments at which the planet's center coincides with the limb of the star. For XO- $1, q=0.0275 \pm 0.0005, r_{*}=1.0 \pm 0.08 \mathrm{R}_{\odot}, m_{*}=1.0 \pm 0.03 \mathrm{M}_{\odot}$, so $\hat{\rho}=1.0 \pm 0.24$ and thus $i=87.7^{\circ} \pm 1.2^{\circ}$.

The radius of the planet $r_{p}$ is approximately proportional to $r_{*}$, because the depth of the transit is approximately equal to $p^{2}$. At a more precise level of approximation, $r_{p} \propto r_{*}^{\kappa}$, where $\kappa$ accounts for limb darkening, which necessitates an increasingly larger $r_{p}$ as $r_{*}$ increases (Equation 3). For various presumed values for $r_{*}$ and $m_{*}$, we minimized $\chi^{2}$ of the R-band data by varying $t_{c}, r_{p}$, and $i$. The region of high likelihood for XO- 1 is described by the 
functional relation,

$$
r_{p} \approx(1.30 \pm 0.03)\left(r_{*} / r_{\odot}\right)^{\kappa} \mathrm{R}_{\mathrm{J}}
$$

where $\kappa \approx 1+0.5 \gamma_{1} L / r_{*}$. As previously defined, $\gamma_{1}$ is the linear limb darkening coefficient, and $L$ is the half chord length of the transit's path across the star. For XO-1 observed in $\mathrm{R}$ band, $\kappa=1$.17. Substituting the best-fit value and uncertainty for the radius of the star into Equation 3, we conclude that the planet's radius ${ }^{5}$ as determined from the R-band light curves is $1.30 \pm 0.11 \mathrm{R}_{\mathrm{J}}$.

Limb darkening is stronger in the blue than the red, so a transit with inclination $i=90^{\circ}$ should be deeper in the blue than the red. The difference in transit depths in B band compared to $\mathrm{R}$ band would be $2 \mathrm{mmag}$ for XO-1b if $i=90^{\circ}$. At the other extreme, a grazing transit, the transit should be shallower in the blue than the red. The transit depth color difference's constraint on inclination requires more accurate photometry than that presented here (cf. Jha et al. 2000).

\section{Discussion}

\subsection{Transit Timing}

Other planets in the XO-1 system could be revealed by their transits or by their perturbations of the radial velocity of XO-1 or on the time of arrival of the transits by XO-1b. We have not observed sufficient numbers of transits by XO-1b with adequate quality to justify an analysis of time-of-arrival perturbations. To motivate such observations, we note their scientific value and present an analysis of the dependence of timing precision on photometric precision.

Transit timing observations would be especially sensitive to planets in orbital resonance(s) with XO-1b (Steffen \& Agol 2005; Holman \& Murray 2005). In principle the error in a measurement of the time of arrival of a transit is proportional to the photometric error per unit time. We estimate that for an idealized transit light curve, with an isosceles trapezoid shape, i.e. with negligible limb darkening, the error in the time of arrival (TOA)

\footnotetext{
${ }^{5}$ Authors unwittingly create ambiguity if they do not define $R_{J}$ explicitly, because various averages of Jupiter's polar radius $R_{p}$ and its equatorial radius $R_{e}$ could be selected for $\mathrm{R}_{\mathrm{J}}$. Because $R_{p} \approx 0.94 R_{e}$ (Mallama et al. 2000), such ambiguity can be significant compared to observational uncertainties; e.g. Knutson et al. (2006) report the radius of HD 209458 with a fractional uncertainty of $1.9 \%$. From reported masses and mean densities, we determined that many authors tacitly define $\mathrm{R}_{\mathrm{J}}=R_{e}=71492 \mathrm{~km}$; hence we have also, for the sake of consistency.
} 
of each transit is

$$
\sigma^{2}(T O A) \approx\left(\frac{\sigma_{m} w}{d \sqrt{N}}\right)^{2}+\left(\frac{\epsilon w}{2 d}\right)^{2}
$$

where $\sigma_{m} / d$ is the dimensionless ratio of the standard deviation of the photometry to the depth of the transit, $N$ is number of observations obtained during ingress and/or egress, $w$ is the time interval between first and second contacts, and $\epsilon$ is a characteristic error due to (uncalibrated) secular variations in gain or atmospheric transparency over a time scale equal to w. Implicit in Equation 4 is that 1) the light curve shape is known, 2) there are sufficient observations to set the level of the light curve's out-of-transit portion precisely, 3) the noise has a normal distribution, and 4) $\mathrm{N} \gg 1$. For example, if $\epsilon=0$ and one can obtain $\sigma_{m}=2 \mathrm{mmag}$ precision per 1-minute observation of a transit with depth $d=20 \mathrm{mmag}$ and duration of ingress $w=20$ minutes, then for an observation of ingress, $N=20$ and the time can be measured with a standard deviation $\sigma(T O A)=27$ seconds. If ingress and egress are both observed, then $N=40$ and $\sigma(T O A)=19$ seconds for the transit as a whole. For $\epsilon= \pm 1$ mmag the measured time of arrival of the transit is shifted by \pm 30 seconds. If there are additional uncalibrated systematics in an observed light curve, then Equation 4 will underestimate the uncertainty of the time of arrival. In particular, if only ingress or egress is observed, then secular trends are more difficult to calibrate and the residual error in calibration, $\epsilon$, will be larger than if many observations are obtained immediately before and after each transit.

Because scintillation is independent of the brightness of the star, the photometric precision, and hence the timing precision of transits observed from the Earth's surface, is not strongly dependent of the brightness of the target star, for stars as bright or brighter than XO-1. If the star is sufficiently faint that the Poisson contribution to noise is much larger than the scintillation, e.g. if the sensor or the spectral filters limit the collecting efficiency, such as in B band, or if spectroscopy is the goal, bright stars and/or large telescopes can have a significant advantage. The Poisson noise for XO-1 observed with a $0.3-\mathrm{m}$ telescope with an R-band filter is $0.15 \%$ (i.e. $\sim 1.5 \mathrm{mmag}$ ) in a 60 -second exposure. Near sea level, typical scintillation for such a telescope and exposure time is 0.9, 1.7, and 2.9 mmag at air masses of 1, 1.5, and 2, respectively (Young 1967; Dravins et al. 1998). For differential photometry, comparison stars comparable in brightness to the target star generally will be nearer to a faint target than a bright target. The latter effect can be important if the nearest suitable comparison star is too distant in angle to observe with the target simultaneously on the same sensor.

We searched for a second planet transiting XO-1 by removing the observations within 0.06 days of minimum light of the known transit. The sensitivity to transits decreases with increasing period, and the a priori likelihood that a planet orbiting XO-1 will transit 
also decreases with period. Due to the measurement errors of the photometry from the $\mathrm{XO}$ cameras and the radial velocities, respectively, we would not have detected a second planet orbiting XO- 1 if its radius $r_{p} \lesssim 0.5 \mathrm{R}_{\mathrm{J}}$ or if its mass $m_{p}$, inclination $i$, and angular frequency, are such that $m_{p} \sin (i)\left(\omega_{2} / \omega_{1}\right)^{1 / 3} \lesssim 0.5 \mathrm{M}_{\mathrm{J}}$, where $\omega_{2} / \omega_{1}$ is the ratio of the angular frequency of the second planet to that of XO-1b. The complementary statement, that we would have detected a second planet if either or both of those inequalities were not true, cannot be definitive in general. In particular, additional planets could orbit XO-1 with periods much longer than that of XO-1b. Monitoring XO-1's radial velocity could reveal additional planets and enable predictions of the approximate times that they might transit.

\subsection{A Triple Star?}

Mandushev et al. (2005) demonstrate that a triple star system (gravitationally bound or an asterism) can exhibit a light curve and a radial velocity curve similar to that of a planet or brown dwarf transiting a single star. The rotational broadening of Mandushev et al's stars, (V $\sin i=34 \mathrm{~km} \mathrm{~s}^{-1}$ for the F0 primary; V $\sin i=15 \mathrm{~km} \mathrm{~s}^{-1}$ for the G0 secondary) produced an oscillating asymmetry in the spectral lines that could be misinterpreted as periodicity in the radial velocity of a single star induced by a dark companion of substellar mass. We performed a simpler form of Mandushev et al's analysis for XO-1 which we summarize here. Our analysis is simpler because XO-1 has narrow spectral lines ( $\mathrm{V} \sin i<3 \mathrm{~km} \mathrm{~s}^{-1}$ ), so perturbations by a stellar companion would be observed as shifts of narrow lines, rather than distortions of broad lines.

Mandushev et al's light curve consists of three parts equal in duration: ingress, flat bottom, egress. Although a transiting planet can produce such a light curve, the a priori probability of the duration of ingress (or egress) being as large or larger than the duration of the transit's middle section, between contacts 2 and 3 , is approximately $1-\sqrt{1-2 r_{p} / r_{*}}$, which is always greater than or equal to $p=r_{p} / r_{*}$, and equals $\sim 10 \%$ for a typical Jovian planet transiting a solar-type star. In practice, we expect such grazing transits to be detected with lower than a priori chance, because their reduced depth and duration both reduce detectability. On the other hand, if $p \geq 0.375$, the probability of ingress (or egress) being at least as long as the middle section of the light curve is $\geq 0.5$; that is, eclipsing binary stars typically exhibit such light curves, whereas transiting planets typically do not. Such probabilistic arguments cannot prove a given system is not a transiting planet, but we have used them to focus our efforts on "steep-edged" U-shaped light curves instead of those with ingress and egress comparable in length to the flat bottom.

For XO-1's light curve, the total duration of the flat bottom is $\sim 5$ times the duration of 
ingress or egress, which implies that the ratio of diameters of the eclipsing bodies must be $\gtrsim 5$. For a G1 V primary $\left(r_{*}=1.0 \mathrm{R}_{\odot}\right)$, an eclipsing stellar companion must have a radius $r_{*} \lesssim 0.2 \mathrm{R}_{\odot}$ to match the duration of ingress relative to the flat bottom, which implies a spectral type no earlier than M5 V. Diluting the transit depth with a second G1 V star in the system would mimic the observed depth $(2 \%)$ without affecting the durations. ${ }^{6}$ However, the radial velocity shifts of a $\mathrm{G} 1 \mathrm{~V}$ star orbited by a $\mathrm{M} \approx 0.1 \mathrm{M}_{\odot}$ companion would be $\sim 10$ $\mathrm{km} \mathrm{s}^{-1}$. For a star with narrow lines like XO-1, velocity shifts of this amplitude would be apparent in our spectra, in contrast to the situation of stars with much broader spectral lines.

Modeling XO-1 as a triple star with a second star fainter than the G1V primary encounters difficulty with the radius of the third star (that eclipses the second star). As the second star contributes a lesser fraction of the total light of the system, we are forced to make the third star an ever larger fraction of the size of the star it eclipses, in order to match the $2 \%$ depth. However, from the light curve we know that the ratio of diameters of the eclipsing bodies must be $\gtrsim 5$.

Modeling XO-1 as a triple star cannot include a secondary star brighter than the observed narrow-lined G1V star, unless the secondary star's spectrum is featureless, perhaps due to great rotational broadening. However, the broadband colors of XO-1 are consistent with a slightly reddened ${ }^{7} T_{\text {eff }}=5750 \mathrm{~K}$ star (Table 2 ).

We conclude the XO-1 transit cannot be caused by a stellar binary diluted by a third star that is either physically bound or along the line of sight, due to the mutual constraints of eclipse depth, eclipse shape, and a single-lined G1V spectrum with $\mathrm{V}$ sin $i<3 \mathrm{~km} \mathrm{~s}^{-1}$.

$\mathrm{XO}-1 \mathrm{~b}$ must be a planet, but the XO-1 system could include a wide-separation stellar companion that is either much fainter than or very similar in spectra to the G1V star. For example, HD 189733 has a faint stellar companion (Bakos et al. 2006b). For XO-1, the possibility of a stellar companion could be tested with infrared adaptive optics on the largest available telescopes.

\footnotetext{
${ }^{6}$ In this hypothetical example, we could replace the M5V star with a planet twice the nominal mass of $\mathrm{XO}-1 \mathrm{~b}$ and twice the latter's projected surface area, and all the observational constraints would be equally well satisfied. However, the density of the planet would be $1 / \sqrt{2}$ times the nominal (single-star hypothesis) value. Such a contrived example seems less plausible than the nominal system of a single star and planet.

${ }^{7}$ In the direction of XO-1, the interstellar extinction $\mathrm{A}_{\mathrm{V}}=0.04 \mathrm{mag}$ as inferred from infrared observations with a $6^{\prime}$ (FWHM) beam (Schlegel, Finkbeiner, \& Davis 1998). We neglect its small $(\lesssim 2 \%)$ effect on the distance and the radius of $\mathrm{XO}-1$.
} 


\subsection{Comparison to other Transiting Planets}

Figure 9 compares the radius and mass of $\mathrm{XO}-1 \mathrm{~b}$ with those of the nine other transiting extrasolar planets as tabulated by Charbonneau et al. (2006). The mean density of XO-1b is $0.51 \pm 0.13 \mathrm{~g} \mathrm{~cm}^{-3}$. The radius of the star XO-1 is not known to sufficient accuracy to assert that its planet XO-1b is truly anomalously large. If the G1 V star XO-1 has the same radius as the Sun, then the planet XO-1b is approximately the size of HD 209458b. The radius of each of these two large planets, $\sim 1.3$ times the radius of Jupiter, is $\sim 20 \%$ larger than theoretical models predict (Table 1 of Bodenheimer et al. 2003). To keep the planet's size large, an additional heat (or power) source is hypothesized deep inside the planet. Knutson et al. (2006 and references therein) summarize three proposed models for that heat source: 1) strong winds on the planet, with the original power source being the incident light of the star, 2) tidal dissipation, presumably due to a second planet forcing the hot Jupiter into an eccentric orbit, and 3) resonances between the spin precession and the orbital precession, a so-called Cassini state. At $0.90 \mathrm{M}_{\mathrm{J}}, \mathrm{XO}-1 \mathrm{~b}$ is $\sim 2$ times more massive than the best-fit correlation of mass and period noted by Mazeh, Zucker, \& Pont (2005), whereas HD 149026b is $\sim 2$ times less massive than the same correlation.

\section{Summary}

The planet XO- $1 \mathrm{~b}$ is the first planet discovered by the XO project. It is similar to other transiting extrasolar planets in its physical characteristics (Section 4.3; Table 3; Figure 9). Of those nine others, HD 209458b has the most similar radius to the nominal radius of XO1b. The host star XO-1 is very similar to the Sun in its physical characteristics (Tables 2, 5, and 6). Equation 2 expresses the inclination of a transiting planet's orbit as a function of its period, the duty cycle of the transit, and the mean density of the star. For transits with imprecisely measured inclinations near $90^{\circ}$, the derived ratio of the radii of the planet and the star is approximately proportional to the stellar radius raised to a power equal to one half the linear limb darkening coefficient (Equation 3). We note the scientific potential

of transit timing and estimate limits to its accuracy. We discuss evidence that disproves the hypothesis that the periodic modulations of the photometry and the radial velocity of XO-1 are due to a triple star system.

The University of Hawaii staff have made the operation on Maui possible; we thank especially Bill Giebink, Les Hieda, Jake Kamibayashi, Jeff Kuhn, Haosheng Lin, Mike Maberry, Daniel O'Gara, Joey Perreira, Kaila Rhoden, and the director of the IFA, Rolf-Peter Kudritzki. 
We thank Lisa Prato, Marcos Huerta, Danielle Best, and Josh Shiode for assistance observing. We acknowledge helpful discussions with Gaspar Bakos, Christopher Burke, Fred Chromey, Ron Gilliland, Leslie Hebb, James McCullough, Margaret Meixner, and Kailash Sahu.

This research has made use of a Beowulf cluster constructed by Frank Summers; the SIMBAD database, operated at CDS, Strasbourg, France; data products from the Two Micron All Sky Survey (2MASS) and the Digitized Sky Survey (DSS); source code for transit light-curves (Mandel \& Agol 2002); and community access to the HET. We thank the support staff of the McDonald Observatory.

$\mathrm{XO}$ is funded primarily by NASA Origins grant NAG5-13130, and in the past has received financial support from the Sloan Foundation, the Research Corporation, the Director's Discretionary Fund of the STScI, and the US National Science Foundation.

\section{REFERENCES}

Alonso, R., et al. 2004, ApJ, 613, L153

Bakos, G. A., et al. 2006a, ApJ, submitted (astroph/0603291).

Bakos, G. A., et al. 2006b, ApJ, submitted (astroph/0602136).

Bessell, M. S. 2000, PASP, 112, 961

Bodenheimer, P., Lin, D. N. C., \& Mardling, R. A. 2001, ApJ, 548, 466

Bodenheimer, P., Laughlin, G., \& Lin, D. N. C. 2003, ApJ, 592, 555

Bouchy, F., et al. 2005a, A\&A, 444, L15

Bouchy, F., Pont, F., Melo, C., Santos, N. C., Mayor, M., Queloz, D., \& Udry, S. 2005b, A\&A, 431, 1105

Brown, T. M. 2003, ApJ, 593, L125

Brown, T. M., Charbonneau, D., Gilliland, R. L., Noyes, R. W., \& Burrows, A. 2001, ApJ, 552,699

Charbonneau, D., Brown, T. M., Burrows, A., \& Laughlin, G. 2006, ArXiv Astrophysics e-prints, arXiv:astro-ph/0603376 
Charbonneau, D., Brown, T. M., Dunham, E. W., Latham, D. W., Looper, D. L., \& Mandushev, G. 2004, AIP Conf. Proc. 713: The Search for Other Worlds, 713, 151

Charbonneau, D., Brown, T. M., Latham, D. W., \& Mayor, M. 2000, ApJ, 529, L45

Charbonneau, D., Brown, T. M., Noyes, R. W., \& Gilliland, R. L. 2002, Detection of an Extrasolar Planet Atmosphere, ApJ, 568, 377

Charbonneau, D., et al. 2005, ApJ, 626, 523

Claret, A. 2000, A\&A, 363, 1081

Cochran, W. 2000, FTS spectrum of I2 Cell HRS3 at 69.9 C., ftp://nsokp.nso.edu/FTS_cdrom/FTS50/001023R0.004

da Silva, R., et al. 2006, A\&A, 446, 717

Demarque, P., Woo, J.-H., Kim, Y.-C., \& Yi, S. K. 2004, ApJS, 155, 667

Deming, D., Seager, S., Richardson, L. J., \& Harrington, J. 2005, Infrared radiation from an extrasolar planet, Nature, 434, 740

Dravins, D., Lindegren, L., Mezey, E. \& Young, A. T. 1998, PASP, 110, 610

Fischer, D. A., \& Valenti, J. 2005, ApJ, 622, 1102

Gray, D. F. 1992, The Observation and Analysis of Stellar Photospheres (2nd ed.; Cambridge: Cambridge Univ. Press)

Gray, D. F. 1994, PASP, 106, 1248

Gray, D. F. \& Brown, K. 2001, PASP, 113, 723

Gray, D. F. \& Johanson, H. 1991, PASP, 103, 439

Henry, G. W., Marcy, G. W., Butler, R. P., \& Vogt, S. S. 2000, ApJ, 529, L41

Høg, E., et al. 2000, A\&A, 355, L27

Henden, A. 2006, Astronomical Society of the Pacific Conference Series, 349, 165

Hinkle, K. H., Joyce, R. R., Sharp, N., \& Valenti, J. A. 2000, Proc. SPIE, 4008, 720

Holman, M. J., \& Murray, N. W. 2005, Science, 307, 1288 
Janes, K. A., Buie, M. W., Bosh, A. S., Clemens, D. P., \& Jackson, J. M. 2005, American Astronomical Society Meeting Abstracts, 207, \#173.11

Jha, S., Charbonneau, D., Garnavich, P. M., Sullivan, D. J., Sullivan, T., Brown, T. M., \& Tonry, J. L. 2000, ApJ, 540, L45

Konacki, M., Torres, G., Sasselov, D. D., \& Jha, S. 2005, ApJ, 624, 372

Knutson, H., Charbonneau, D., Noyes, R. W., Brown, T. M., \& Gilliland, R. L. 2006, ArXiv Astrophysics e-prints, arXiv:astro-ph/0603542

Landolt, A. U. 1992, AJ, 104, 340

Mallama, A., Krobusek, B. A., Collins, D. F., Nelson, P., \& Park, J. 2000, Icarus, 144, 99

Mandel, K., \& Agol, E. 2002, ApJ, 580, L171

Mandushev, G., et al. 2005, ApJ, 621, 1061

Mazeh, T., Zucker, S., \& Pont, F. 2005, MNRAS, 356, 955

McCullough, P. R., Stys, J. E., Valenti, J. A., Fleming, S. W., Janes, K. A., \& Heasley, J. N. 2005, PASP, 117, 783

Naeye, R. 2004, S\&T, 108, 18

Nelder, J. A. \& Mead, R. 1965, Computer J., 7, 308

O’Donovan, F. T., Charbonneau, D., \& Kotredes, L. 2004, AIP Conf. Proc. 713: The Search for Other Worlds, 713, 169

Robinson, S. E., Strader, J., Ammons, S. M., Laughlin, G., \& Fischer, D. 2006, ApJ, 637, 1102

Sato, B., et al. 2005, ApJ, 633, 465

Schlegel, D. J., Finkbeiner, D. P., \& Davis, M. 1998, ApJ, 500, 525

Seagroves, S., Harker, J., Laughlin, G., Lacy, J., \& Castellano, T. 2003, PASP, 115, 1355

Silva, A. V. R. 2003, ApJ, 585, L147

Skrutskie, M. F., et al. 2006, AJ, 131, 1163

Steffen, J. H., \& Agol, E. 2005, MNRAS, 364, L96 
Tull, R. G. 1998, Proc. SPIE, 3355, 387

Tull, R. G., MacQueen, P. J., Sneden, C., \& Lambert, D. L. 1995, PASP, 107, 251

Valenti, J. A., \& Fischer, D. A. 2005, ApJS, 159, 141

Valenti, J. A., \& Piskunov, N. 1996, A\&AS, 118, 595

VandenBerg, D. A., \& Clem, J. L. 2003, AJ, 126, 778

Vidal-Madjar, A., et al. 2004, ApJL, 604, L69

Vidal-Madjar, A., Lecavelier des Etangs, A., Désert, J.-M., Ballester, G. E., Ferlet, R., Hébrard, G., \& Mayor, M. 2003, Nature, 422, 143

Wallace, L., Hinkle, K., \& Livingston, W. 1998, An atlas of the spectrum of the solar photosphere from 13,500 to 28,000 cm-1 (3570 to 7405 A), Publisher: Tucson, AZ: National Optical Astronomy Observatories

Wyse, R. F. G., \& Gilmore, G. 1995, AJ, 110, 2771

Young, A. T. 1967, AJ, 72, 747 
Table 1. All-sky Photometric Magnitudes

\begin{tabular}{ccccc}
\hline \hline Star $^{\mathrm{a}}$ & $\mathrm{B}$ & $\mathrm{V}$ & $\mathrm{R}_{\mathrm{C}}$ & $\mathrm{I}_{\mathrm{C}}$ \\
\hline $\mathrm{XO}-1$ & 11.85 & 11.19 & 10.806 & 10.43 \\
1 & 13.85 & 12.85 & 12.282 & 11.76 \\
2 & 16.31 & 14.93 & 14.139 & 13.41 \\
3 & 12.36 & 11.54 & 11.046 & 10.56 \\
4 & 14.31 & 13.65 & 13.313 & 12.93 \\
5 & 9.99 & 9.54 & 9.261 & 8.97 \\
6 & 12.42 & 11.29 & 10.680 & 10.11 \\
7 & 11.85 & 10.86 & 10.330 & 9.81 \\
8 & 15.08 & 14.28 & 13.803 & 13.28 \\
\hline
\end{tabular}

a) Stars are identified in Figure 1.

Table 2. The Star XO-1

\begin{tabular}{lcl}
\hline \hline Parameter & Value & Reference \\
\hline RA (J2000.0) & $16^{h} 02^{m} 11^{s} .84$ & $\mathrm{a}, \mathrm{b}$ \\
Dec (J2000.0) & $+28^{\circ} 10^{\prime} 10^{\prime \prime} .4$ & $\mathrm{a}, \mathrm{b}$ \\
$V, V_{T}$ & $11.19 \pm 0.03,11.323$ & $\mathrm{c}, \mathrm{b}$ \\
$B-V, B_{T}-V_{T}$ & $0.66 \pm 0.05,0.692$ & $\mathrm{c}, \mathrm{b}$ \\
$V-R_{C}$ & $0.38 \pm 0.04$ & $\mathrm{c}$ \\
$R_{C}-I_{C}$ & $0.38 \pm 0.04$ & $\mathrm{c}$ \\
$J$ & 9.939 & $\mathrm{~d}$ \\
$J-H$ & 0.338 & $\mathrm{~d}$ \\
$H-K$ & 0.074 & $\mathrm{~d}$ \\
Spectral Type & $\mathrm{G} 1 \mathrm{~V}$ & $\mathrm{c}$ \\
$\mathrm{d}$ & $200 \pm 20 \mathrm{pc}$ & $\mathrm{c}$ \\
$\left(\mu_{\alpha}, \mu_{\delta}\right)$ & $02041-01657$ & $\mathrm{~b}$ \\
GSC & $-19.7 \pm 2.0,15.0 \pm 1.9) \mathrm{mas} \mathrm{yr}^{-1}$ & $\mathrm{a}$ \\
\hline
\end{tabular}

References:

a) SIMBAD

b) Tycho-2 Catalogue, Hog et al (2000)

c) this work

d) 2MASS, Skrutskie e al. (2006) 
Table 3. The Planet XO-1b

\begin{tabular}{lcl}
\hline \hline Parameter & Value & Notes \\
\hline$P$ & $3.941534 \pm 0.000027 \mathrm{~d}$ \\
$t_{c}$ & $2453808.9170 \pm 0.0011(\mathrm{HJD})$ \\
$K$ & $116 \pm 9 \mathrm{~m} \mathrm{~s}^{-1}$ \\
$R_{\mathrm{p}} / R_{\mathrm{s}}$ & $(1.30 \pm 0.04) \times \mathrm{R}_{\mathrm{J}} / \mathrm{R}_{\odot}$ & $\mathrm{a}$ \\
$a$ & $0.0488 \pm 0.0005 \mathrm{~A} . \mathrm{U}$. & $\mathrm{b}$ \\
$i$ & $87.7 \pm 1.2 \mathrm{deg}$ & $\mathrm{b}, \mathrm{c}$ \\
$M_{\mathrm{p}}$ & $0.90 \pm 0.07 \mathrm{M}_{\mathrm{J}}$ & $\mathrm{b}, \mathrm{d}$ \\
$R_{\mathrm{p}}$ & $1.30 \pm 0.11 \mathrm{R}_{\mathrm{J}}$ & $\mathrm{a}, \mathrm{b}, \mathrm{c}$ \\
\hline
\end{tabular}

Notes:

a) $\mathrm{R}_{\mathrm{J}}=71492 \mathrm{~km}$, i.e. the equatorial radius of Jupiter

b) for $M_{*}=1.0 \pm 0.03 \mathrm{M}_{\odot}$

c) for $R_{*}=1.0 \pm 0.08 \mathrm{R}_{\odot}$

d) $\mathrm{M}_{\mathrm{J}}=1.8988 \mathrm{e} 27 \mathrm{~kg}$

Table 4. Radial Velocity Shifts

\begin{tabular}{cccc}
\hline \hline Julian Date & $\begin{array}{c}\text { Radial Velocity } \\
\text { Shift }\left[\mathrm{m} \mathrm{s}^{-1}\right]\end{array}$ & $\begin{array}{c}\text { Uncertainty } \\
(1 \sigma)\left[\mathrm{m} \mathrm{s}^{-1}\right]\end{array}$ & Telescope \\
\hline 2453770.947 & -99 & 63 & HJS 2.7-m \\
2453771.945 & 93 & 70 & HJS \\
2453773.924 & -107 & 63 & HJS \\
2453775.936 & 122 & 64 & HJS \\
& & & \\
2453779.941 & 87 & 15 & HET 11-m \\
2453796.894 & 41 & 17 & HET \\
2453799.884 & 112 & 14 & HET \\
2453801.887 & -111 & 15 & HET \\
2453815.848 & 128 & 21 & HET \\
2453824.815 & -34 & 18 & HET \\
\hline
\end{tabular}


Table 5. Results of the SME Analysis

\begin{tabular}{ccc}
\hline \hline Parameter & Mean & $\begin{array}{c}\text { Precision } \\
(1 \sigma)\end{array}$ \\
\hline$T_{\text {eff }}[K]$ & 5750 & 13 \\
$\log g\left[\mathrm{~cm} \mathrm{~s}^{-2}\right]$ & 4.53 & 0.065 \\
$\mathrm{~V} \mathrm{sin} i\left[\mathrm{~km} \mathrm{~s}^{-1}\right]$ & 1.11 & 0.67 \\
{$[\mathrm{M} / \mathrm{H}]$} & 0.058 & 0.040 \\
{$[\mathrm{Na} / \mathrm{H}]$} & -0.051 & 0.041 \\
{$[\mathrm{Si} / \mathrm{H}]$} & -0.043 & 0.038 \\
{$[\mathrm{Ti} / \mathrm{H}]$} & -0.000 & 0.040 \\
{$[\mathrm{Fe} / \mathrm{H}]$} & 0.015 & 0.040 \\
{$[\mathrm{Ni} / \mathrm{H}]$} & -0.060 & 0.021 \\
{$[\mathrm{Si} / \mathrm{Fe}]$} & -0.058 & 0.056 \\
\hline
\end{tabular}

Table 6. Spectroscopically Derived Stellar parameters

\begin{tabular}{lccc}
\hline \hline Parameter & @ $180 \mathrm{pc}$ & @ $200 \mathrm{pc}$ & @ 220 pc \\
\hline \multirow{2}{*}{ Mass $\left[\mathrm{M}_{\odot}\right]$} & 0.98 & 0.97 & 0.97 \\
& 1.01 & 1.00 & 1.00 \\
& 1.03 & 1.02 & 1.02 \\
& & & \\
Radius $\left[\mathrm{R}_{\odot}\right]$ & 0.92 & 0.96 & 1.04 \\
& 0.95 & 1.00 & 1.09 \\
& 0.98 & 1.06 & 1.15 \\
& & & \\
$\log (\mathrm{g})\left[\mathrm{cm} \mathrm{s}{ }^{-2}\right]$ & 4.44 & 4.38 & 4.31 \\
& 4.52 & 4.43 & 4.35 \\
& & & 4.40 \\
Age $[\mathrm{Gyr}]$ & 0.65 & 2.48 & 5.54 \\
& 2.30 & 4.74 & 6.93 \\
& 4.42 & 6.63 & 8.01
\end{tabular}

For each parameter, the middle row is the maximum likelihood value, and the values in the rows above and below span the $68 \%$ likelihood of the probability distributions (cf. Figure 7). The three columns correspond to three assumed distances for XO-1. 


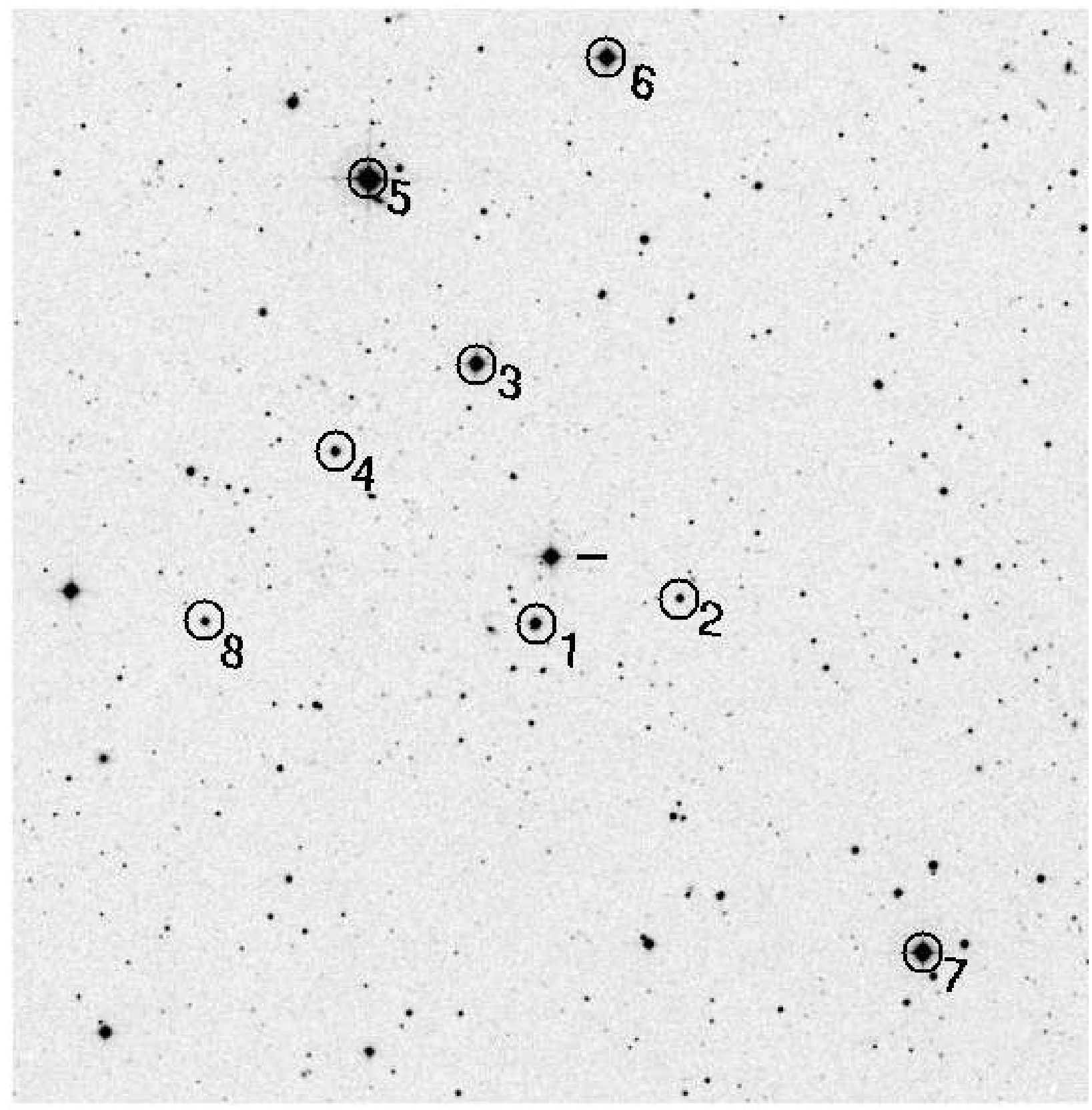

Fig. 1. - XO-1 is centered, indicated by the hash mark. Stars from Table 1 are circled. North is up; East to the left. The DSS image, digitized from a POSSII-F plate with a IIIaF emulsion and an RG610 filter, subtends $19^{\prime}$ of declination. 


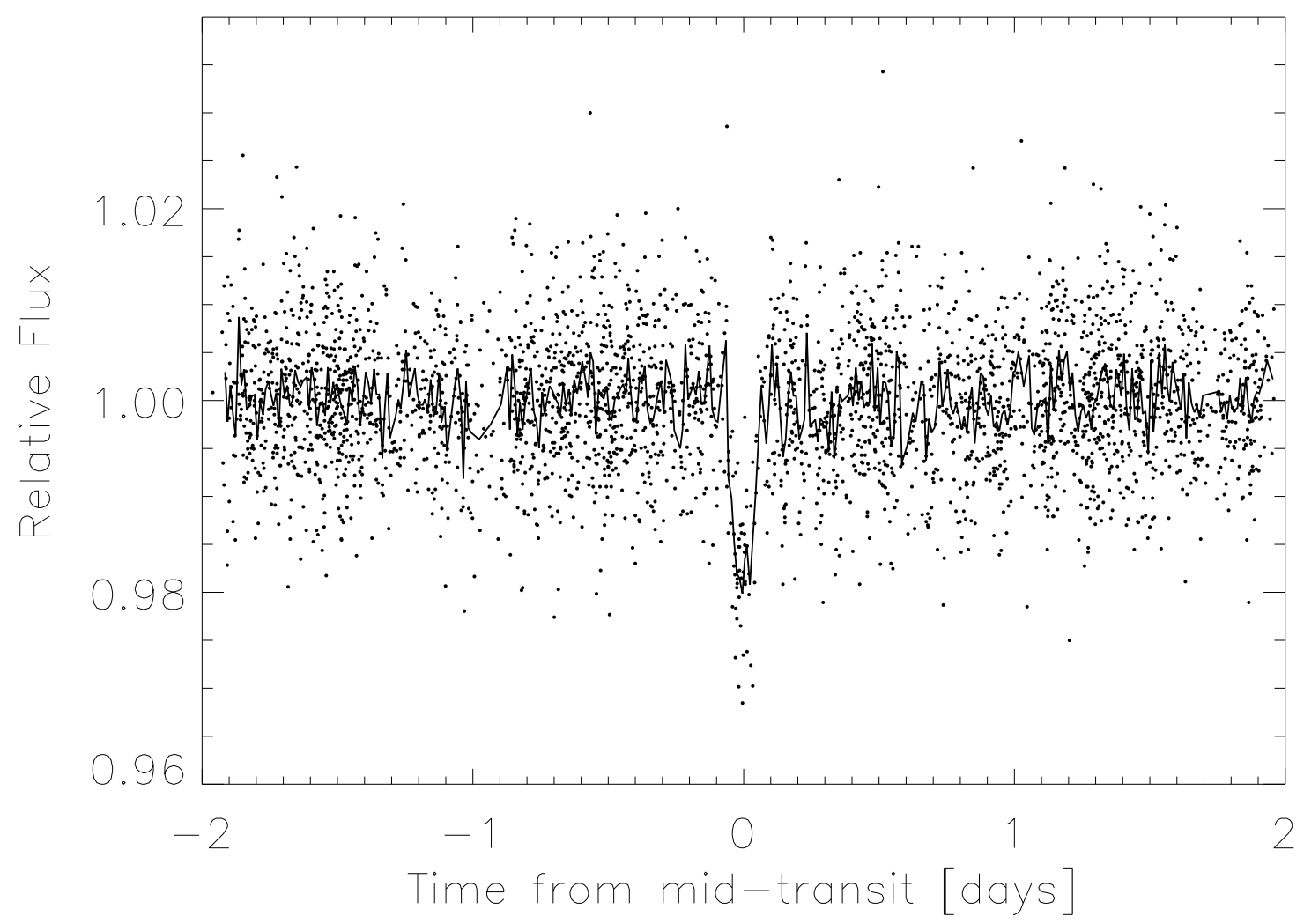

Fig. 2.- More than 3000 individual observations of XO-1 by the two XO cameras over two seasons 2004 and 2005 are shown wrapped and phased according to the ephemeris of Equation 1 and averaged in 0.01-day bins (line). From these data we identified the star as a candidate for more refined photometry with other telescopes at epochs of expected transits (Figure 3). 


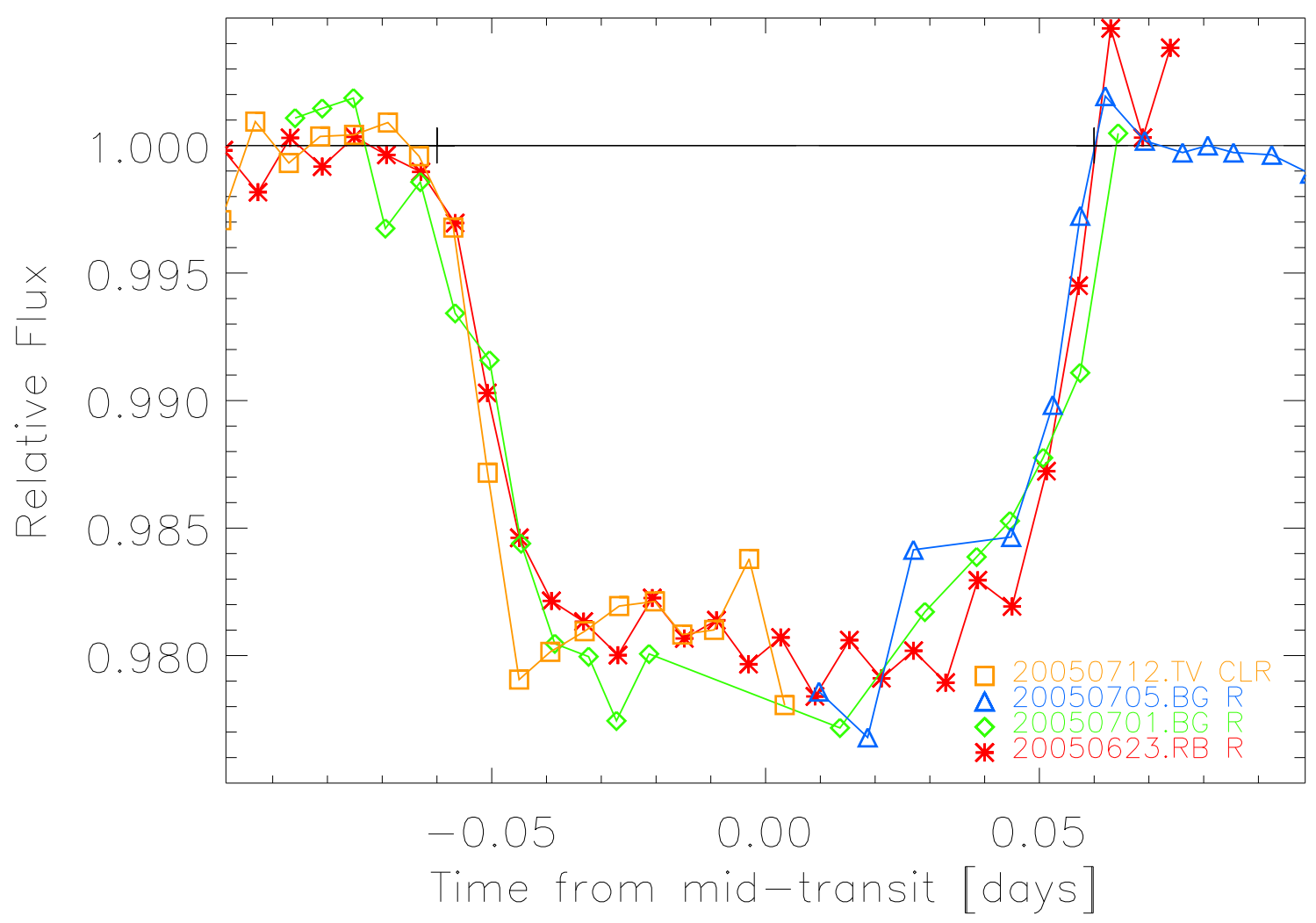

Fig. 3.- Time series photometry of XO-1 from 2005, with dates, observers, and filters indicated. The observations have been averaged in 0.006-day bins. The figure is in color in the electronic edition. 


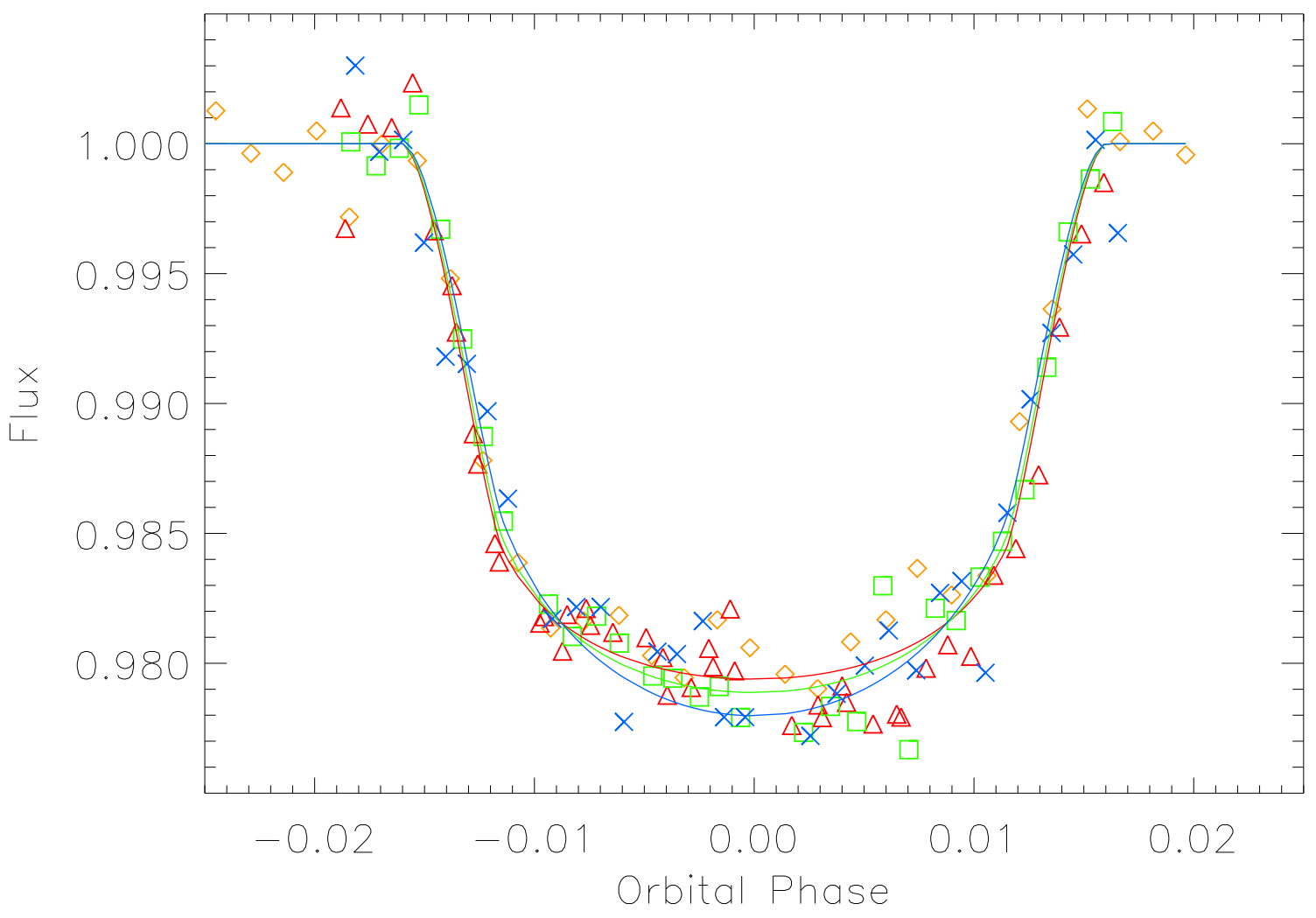

Fig. 4.- Time series photometry of XO-1 from the Mar 14, 2006 transit plotted on the same scale as Figure 3. We fit the R-band data [diamonds (0.3-m telescope; 0.006 day bins) and triangles (1.8-m telescope; 0.002-day bins)] to determine the system parameters for XO-1. Although the shape of the R-band light curve provides the better constraint on the inclination, the V-band (squares) and B-band (crosses) data (both 1.8-m telescope) appear to be slightly deeper and narrower than the R-band, as expected for a near-central $\left(i \approx 90^{\circ}\right)$ transit of a limb-darkened star. The solid lines are models appropriate for each filter (color coded in the electronic edition as $\mathrm{B}=$ blue, $\mathrm{V}=$ green, $\mathrm{R}=\mathrm{red}$ ) with $m_{*}=1.00 \mathrm{M}_{\odot}, r_{*}=1.00$ $\mathrm{R}_{\odot}, \log g=4.53, T_{\text {eff }}=5750 \mathrm{~K}, r_{p}=1.30 \mathrm{R}_{\mathrm{J}}, i=87.7^{\circ}$. 

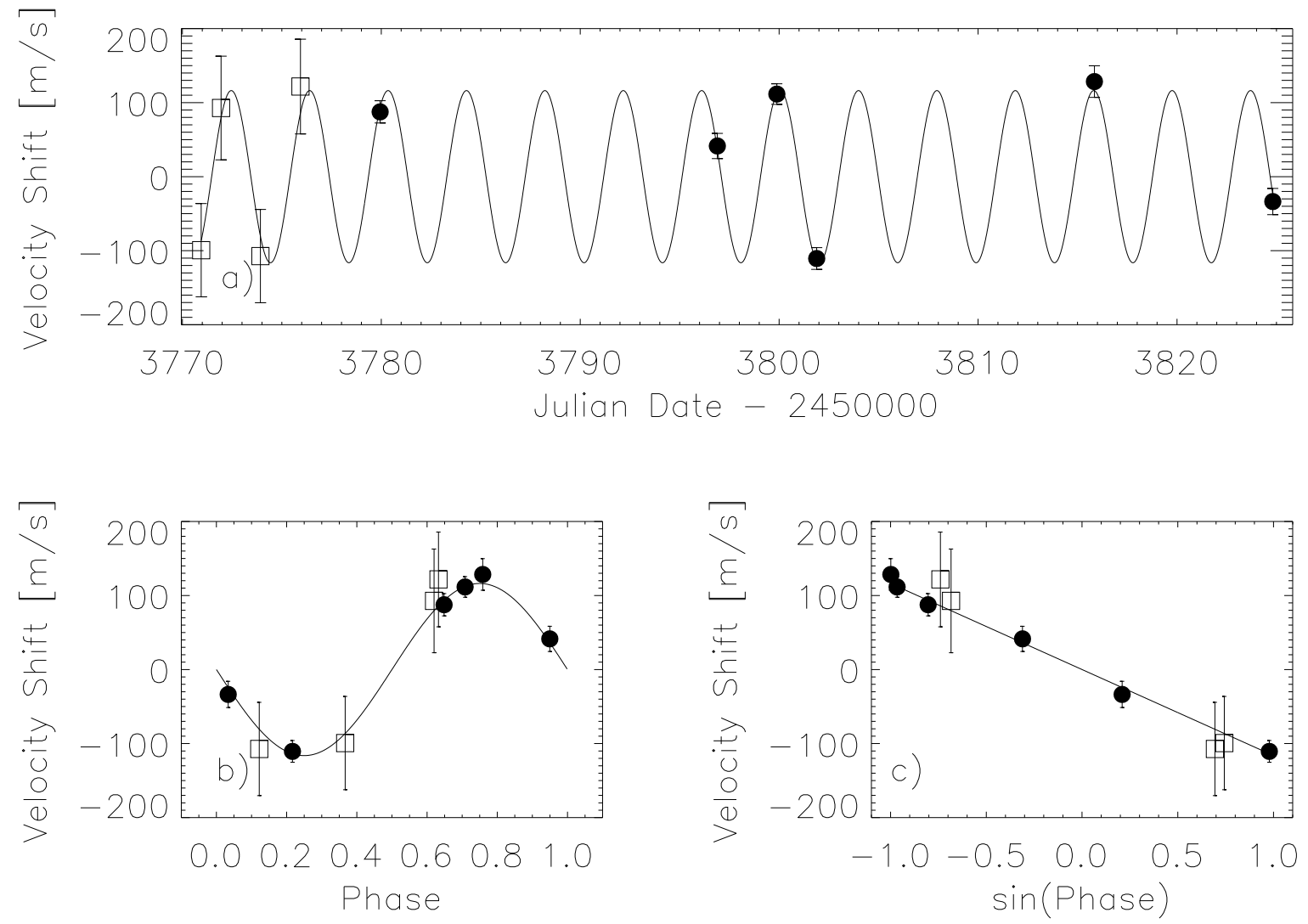

Fig. 5. - a) The radial velocity of XO-1 oscillates sinusoidally with a semi-amplitude $\mathrm{K}=$ $116 \pm 9 \mathrm{~m} \mathrm{~s}^{-1}$, implying XO-1b's mass is $0.90 \pm 0.07 \mathrm{M}_{\mathrm{J}}$. b) The period and phase of the radial velocities were fixed at values determined by the transits. The mean stellar radial velocity with respect to the solar system's barycenter has been subtracted. In order to determine K, we used only the HET data calibrated with an iodine absorption cell (filled circles). The data obtained with the 2.7-m HJS telescope and calibrated with ThAr emission lines (open squares) are for illustration only. We subtracted mean radial velocities separately from the HET and HJS data. Because the data (filled circles and open squares) are from independent observers, telescopes, calibration sources, and analyses, we are confident that the radial velocity oscillation is genuine. c) In this representation of the data, a circular orbit yields a straight line of slope $-\mathrm{K}$. 

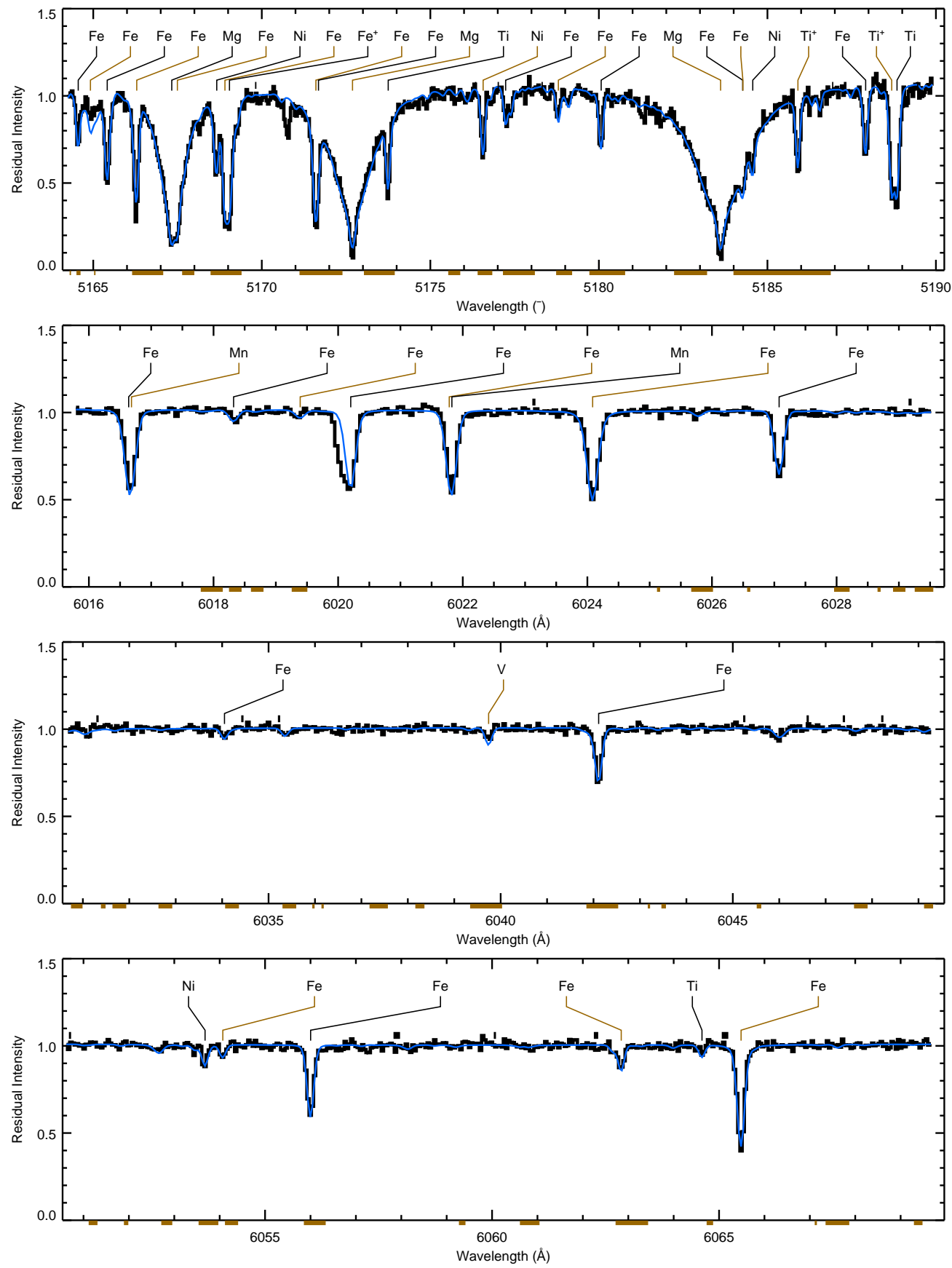

Fig. 6.- The mean spectrum of XO-1 as observed (black histogram) and modeled with SME (curve, colored blue in the electronic edition) in the region of the $\mathrm{Mg} \mathrm{b}$ triplet. Labels note the elements responsible for the indicated spectral lines. Intermittent line segments (tan) beneath the horizontal axis indicate wavelength intervals used to constrain the spectroscopic parameters. Very short and intermittent line segments (black) immediately above the spectrum indicate wavelength intervals used to constrain the continuum fit. 

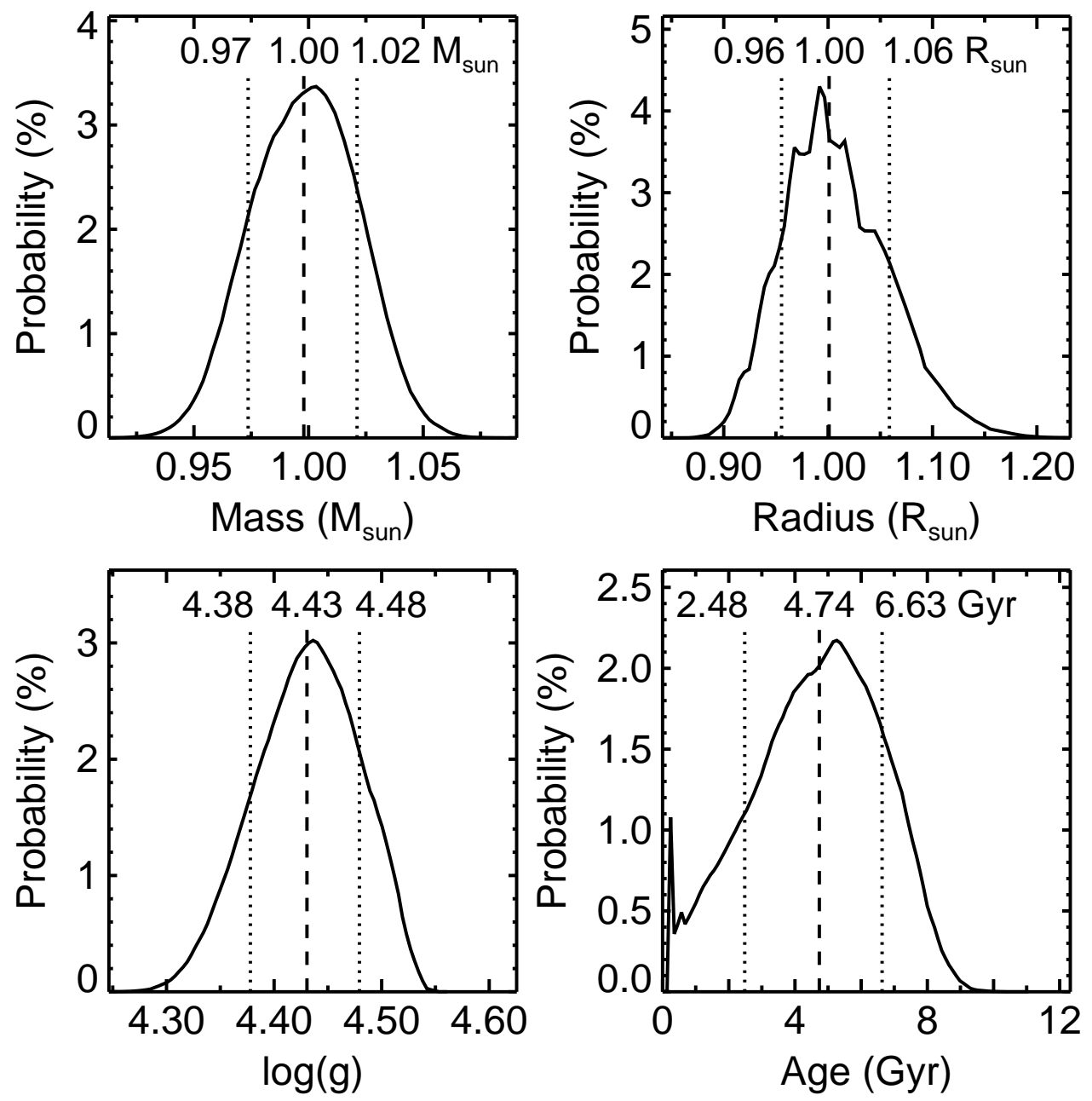

Fig. 7.- Distributions for four stellar parameters derived from the SME analysis (see text) for a distance of 200 pc. The values of the mean and limits containing $\pm 34 \%$ of the distribution from the mean are annotated on the figures and listed in Table 6 along with corresponding values for distances of $180 \mathrm{pc}$ and $220 \mathrm{pc}$. 


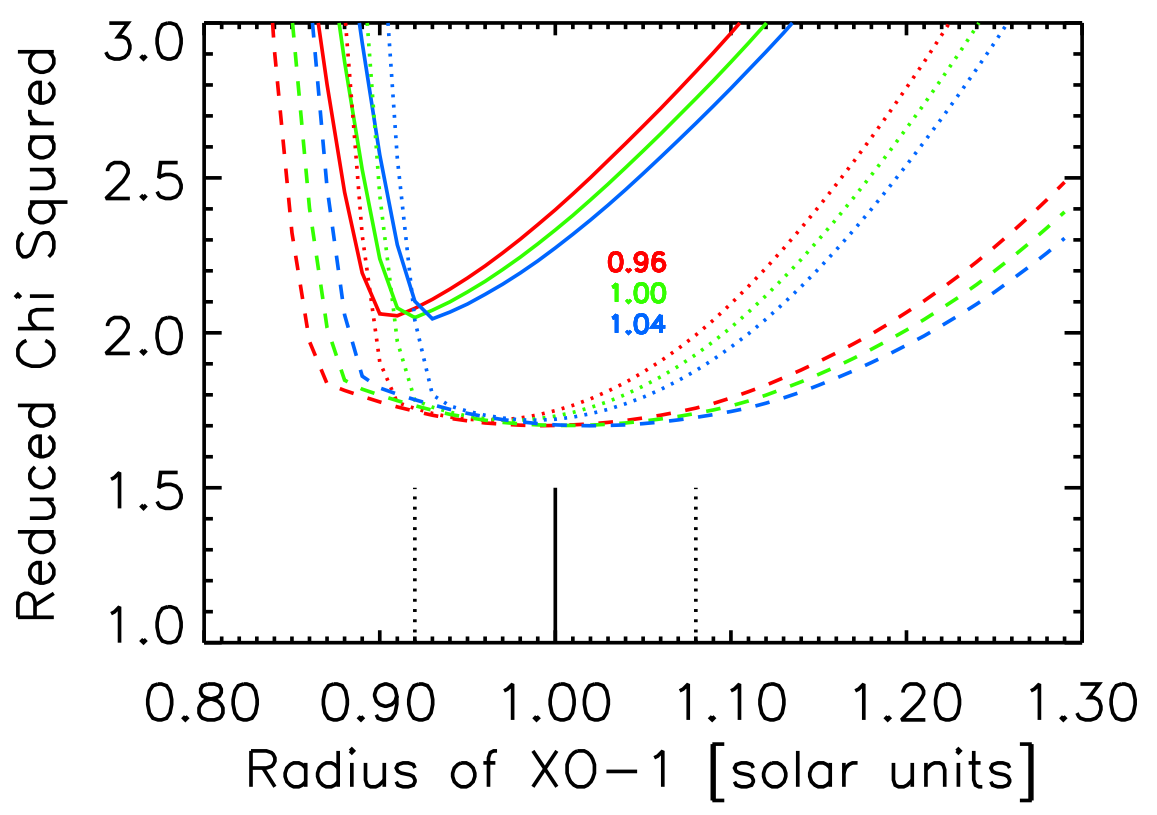

Fig. 8. - The reduced $\chi^{2}$ of light curve models rises away from $\sim 1$ solar radius, more steeply at smaller radii than larger radii because the cosine of the inclination cannot exceed unity (cf. Equation 2). Three independent observations of two transits are shown as solid, dotted, and dashed lines. The nearly coincident lines correspond to $m_{*}=0.96,1.0$, and $1.04 \mathrm{M}_{\odot}$ (colored in the electronic edition). Because the mean stellar density is the critical parameter (Equation 2), at a specific $\chi^{2}$, the more massive models require larger stellar radii. The vertical solid line at $r_{*}=1 \mathrm{R}_{\odot}$ is the maximum likelihood value from the $\mathrm{SME}$ analysis (Table 6); the vertical dotted lines are the corresponding 1- $\sigma$ limits. 


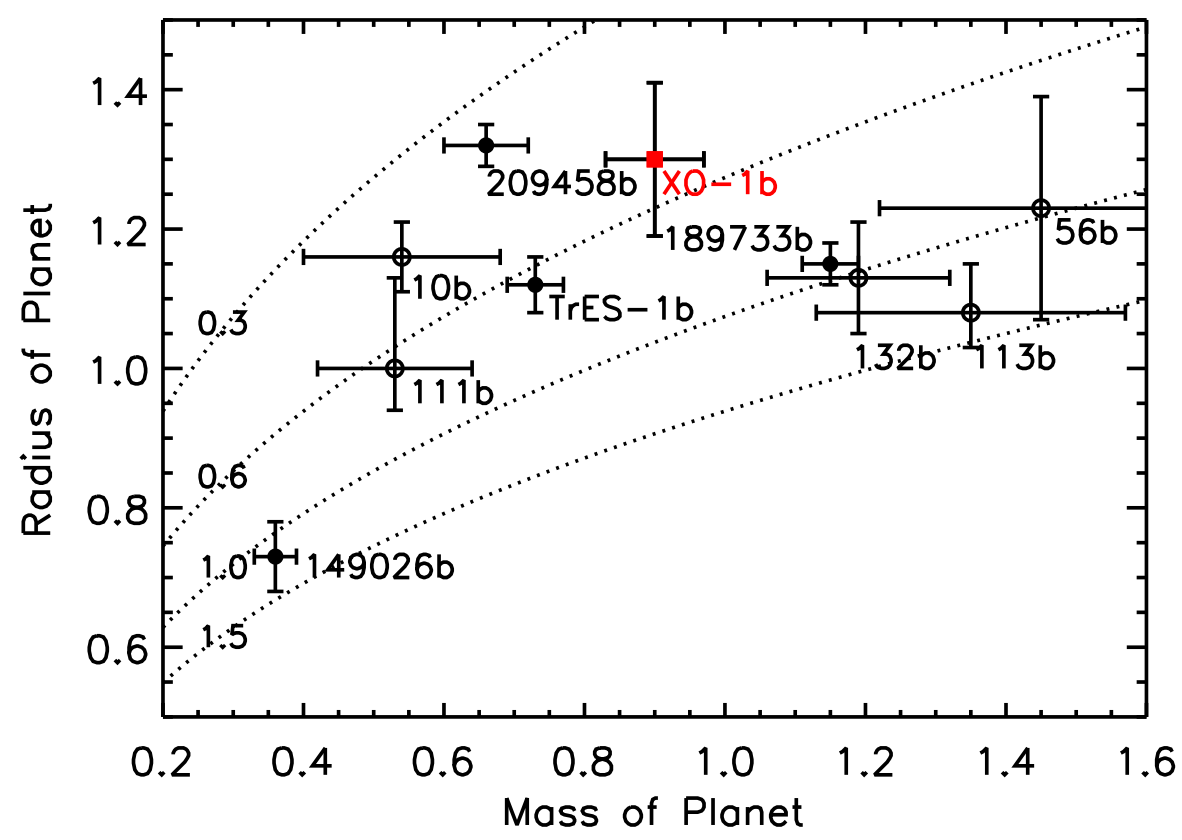

Fig. 9. - To this plot of the radii and masses of transiting extrasolar planets in Jovian units (cf. footnotes to Table 3), we have added XO-1b (red square) to the latest data for the other nine compiled by Charbonneau et al. (2006). Filled symbols are stars brighter than V=12. Open circles represent OGLE planets $(\mathrm{V} \sim 15)$. Lines of constant mean density are labeled in $\mathrm{g} \mathrm{cm}^{-3}$. 